Trauma Berufskrankh $2016 \cdot[$ Suppl 4]: 18:S359-S363

DOI 10.1007/s10039-016-0131-z

Online publiziert: 18. April 2016

(c) Springer-Verlag Berlin Heidelberg 2016

CrossMark

Stefan Simmel · Sabine Drisch · Peter Augat · Volker Bühren

BG-Unfallklinik Murnau, Murnau, Deutschland

\title{
Indikationsprüfung neuer Prothesenpassteile bei Oberschenkelamputierten
}

Hilfsmitteln zu versorgen, die aufgrund des Gesundheitsschadens erforderlich sind. Diese sollen eine drohende Behinderung abwenden, ausgefallene Körperfunktionen ersetzen, beeinträchtigte ausgleichen und die Auswirkungen im medizinischen, beruflichen, schulischen und sozialen Bereich erleichtern.

Dem Unfallversicherungsträger ist hinsichtlich der Art und des Umfanges der Versorgung mit Hilfsmitteln ein Auswahlermessen eingeräumt, soweit nicht die VO 73 (Verordnung über die orthopädische Versorgung Unfallverletzter vom 18.07.1973) eine abschließende Regelung trifft. Die Ausübung des Ermessens hat sich an dem „mit allen geeigneten Mitteln“ anzustrebenden

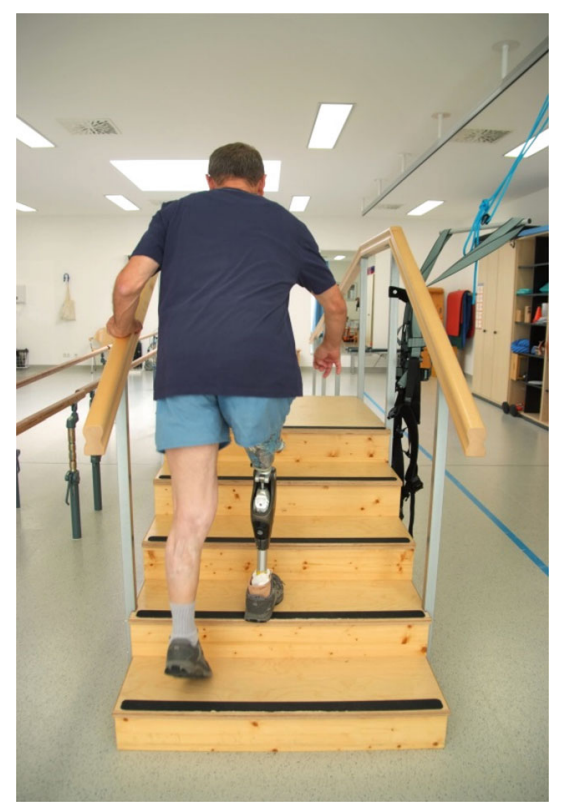

Abb. 1 \ Überprüfung des Kriteriums „Sicherheit" beim Treppengehen
Rehabilitationsziel auszurichten. Dabei sind Art und Schwere des Gesundheitsschadens, die persönlichen, familiären, beruflichen und schulischen Verhältnisse der Versicherten, ihr Bedarf, ihre Leistungsfähigkeit, die örtlichen Verhältnisse sowie ihre angemessenen Wünsche $\mathrm{zu}$ berücksichtigen ( $\$ 33$ SGB I). Zur Bereitstellung der Hilfsmittel ist der konkrete Bedarf der Versicherten durch den Unfallversicherungsträger festzustellen.

\section{Feststellung des Hilfsmittel- bedarfes}

Für die Bedarfsfeststellung ist die medizinische Diagnose nicht allein maßgebend. In Orientierung am biopsychosozialen

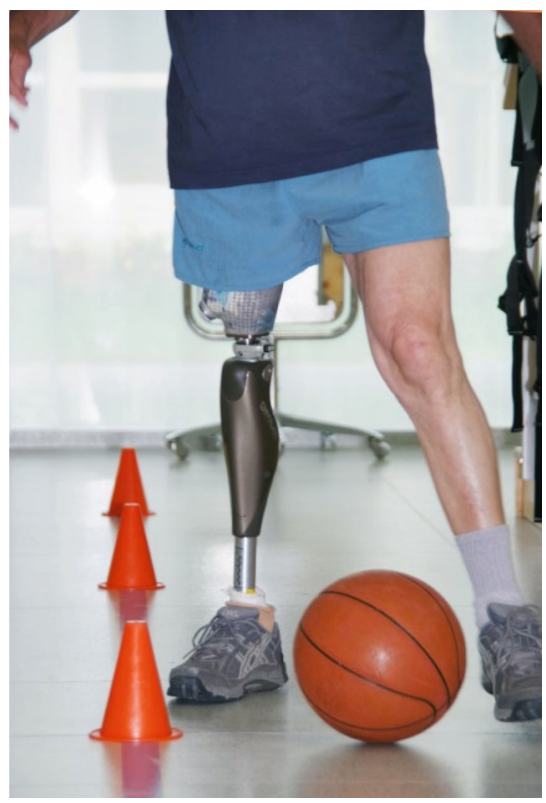

Abb. $2 \Delta$ Überprüfung des Kriteriums "geteilte Aufmerksamkeit" beim Fußballslalom durch Pylonen 


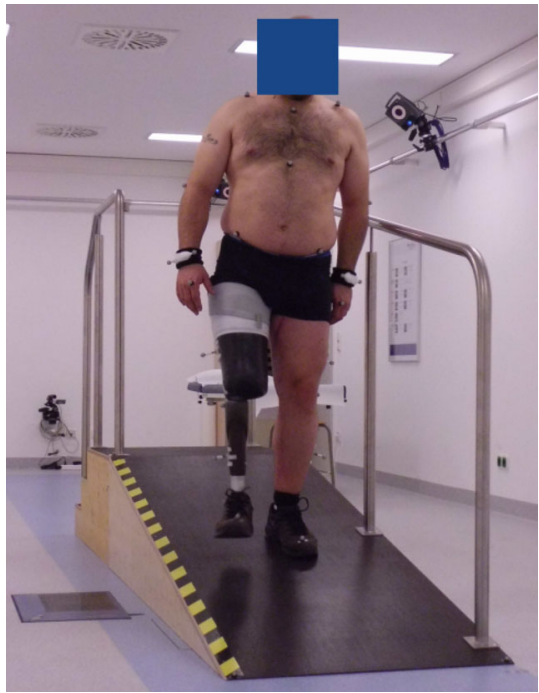

Abb. 3 \& Ganganalyse mit VICON-System beim Hinabgehen einer Schräge

Modell der ICF (Internationale Klassifikation der Funktionsfähigkeit, Behinderung und Gesundheit) ist daneben immer auch eine Gesamtbetrachtung der funktionellen/strukturellen Schädigungen sowie der Beeinträchtigungen und verbliebenen Ressourcen im Bereich der Aktivitäten und Teilhabe erforderlich. Der Bedarf, die Fähigkeit zur Nutzung sowie die Prognose und das Ziel einer Hilfsmittelversorgung sind auf der Grundlage realistischer und alltagsrelevanter Anforderungen zu ermitteln. Dabei sind die individuellen Kontextfaktoren in Bezug auf die Person und die Umwelt als Voraussetzung für die angestrebten Rehabilitations- und Teilhabeziele zu berücksichtigen [2].

Gemäßder ständigen Rechtsprechung des Bundessozialgerichts fällt auch eine hochpreisige Passteilversorgung gegenüber einer konventionellen Prothetik in die Leistungspflicht des Kostenträgers, wenn sich „wesentliche“ Gebrauchsvorteile durch das neue Hilfsmittel ergeben. Dies ist dann der Fall, wenn sich die Gebrauchsvorteile allgemein im Alltagsleben auswirken, sich also nicht bloß auf einen besseren Komfort im Gebrauch oder eine bessere Optik beschränken. „Wesentliche“ Gebrauchsvorteile wären beispielsweise eine weitgehende Annäherung an ein natürliches Gangbild oder eine erhebliche Reduzierung der Sturz-

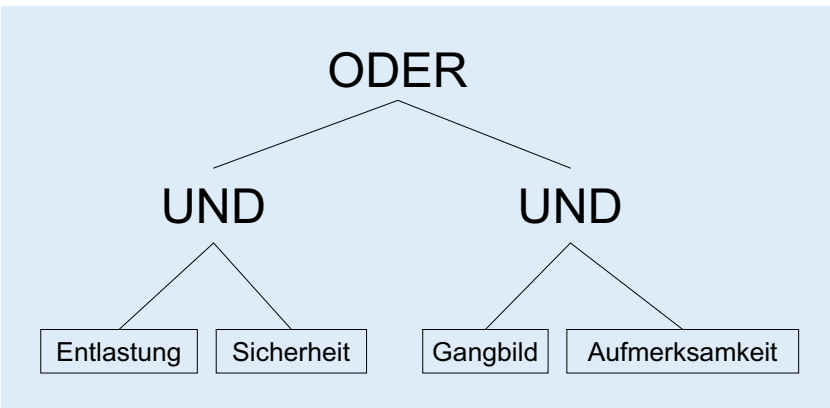

Abb. $4<$ Logi-
scher Baum zum
Entscheidungs-
modell von Kuhr
[4]: (Entlastung
$\wedge$ Sicherheit)
$V$ (Aufmerksam-
keit $\Lambda$ Gangbild)

gefahr beim Gehen auf unebenen Untergründen und auf Treppen.

Die Hilfsmittel sollen dem allgemein anerkannten Stand der technischen Entwicklung entsprechen. Der Patient muss sich dabei nicht darauf verweisen lassen, der bisher verwendete Versorgungsstandard sei ausreichend. Dies gilt auch dann, wenn das bisher genutzte Hilfsmittel noch intakt ist. Kommt ein verbessertes Hilfsmittel auf den Markt und hat es für den Anwender Gebrauchsvorteile, die sich auf seinen gesamten Alltag auswirken, so besteht ein Versorgungsanspruch auf das neue Hilfsmittel (Urteil des Bundessozialgerichts vom 16.09.2004, Az. B 3 KR 2/04 R). Diese Frage stellt sich insbesondere durch die Entwicklung elektronischer Steuerungskonzepte in Prothesenkniegelenken, die in der Regel einen weitergehenden Behinderungsausgleich ermöglichen.

\section{》) Elektronische Steuerungs- konzepte ermöglichen einen weitergehenden Behinderungs- ausgleich}

In der Praxis kann es für den Sachbearbeiter der Unfallversicherung schwierig sein, die Notwendigkeit eines Hilfsmittels und die getroffene Auswahl aus einer Vielzahl zur Verfügung stehender Hilfsmittel nachzuvollziehen. Hat er begründete Zweifel an der Erfordernis oder auch an der Zweckmäßigkeit oder Wirtschaftlichkeit des Versorgungsvorschlages, ist es sinnvoll, zusätzliche Informationen einzuholen und/oder die Verordnung in einem Kompetenzzentrum überprüfen $\mathrm{zu}$ lassen und medizinischen sowie technischen Sachverstand hinzuzuzie- hen. Vor jeder erstmaligen Bewilligung, einer größeren Instandsetzung oder Ersatzbeschaffung eines Hilfsmittels soll deshalb ein sachverständiger Arzt zugezogen werden, wenn es wegen der Art der Versorgung erforderlich ist.

Aber auch für den erfahrenen Arzt ist die Feststellung der Notwendigkeit einer teureren und fraglich besseren Prothesenversorgung häufig nicht einfach. Verschiedene Kriterien können zur Bewertung herangezogen werden. Dabei sind die klinische Beobachtung bzw. die subjektive Meinung des Versicherten nur ein Bestandteil der Entscheidungsfindung. Objektive Messungen und Alltagstests sind erforderlich, um die Indikationsstellung zur Neuversorgung nachvollziehbar zu machen. Die BGU Murnau bietet mit der im Folgenden beschriebenen Indikationsprüfung ein Assessment an, das erforderlich wird bei den Fällen, bei denen sich aus der Diagnose oder der Problemdefinition durch den Patienten und den Arzt nicht eindeutig Art und Umfang der Hilfsmittelversorgung bestimmen lassen oder bei einer geplanten Veränderung ein wesentlicher Gebrauchsvorteil des neuen Hilfsmittels für den Kostenträger nicht sofort erkennbar ist.

Die Frage, die sich immer stellt, lautet: Kann der Amputierte die technischen Möglichkeiten des neuen Passteils tatsächlich nutzen und hat er dadurch Gebrauchsvorteile in seinem Alltag?

\section{Ziele der Indikationsprüfung}

Die Indikationsprüfung neuer Prothesenpassteile der BGU Murnau dient dazu, für den Sachbearbeiter/Rehamanager folgende relevante Informationen $\mathrm{zu}$ sammenzutragen, auf deren Basis dann 
die Entscheidung bezüglich der Versorgung mit einem „besseren“, in der Regel teureren Passteil getroffen werden kann:

- Diagnose(n),

- die für die Hilfsmittelversorgung relevanten Beeinträchtigungen von Strukturen, Funktionen, Aktivitäten und Teilhabe nach ICF,

- relevante Kontextfaktoren, insbesondere Ressourcen, Förderfaktoren und Barrieren,

- Vorgeschichte (auch bisherige Hilfsmittelversorgung),

- Bedarf, Bedürfnisse, Wünsche und Anregungen des Betroffenen,

- Möglichkeiten zur Verbesserung von Funktionen, Aktivitäten und Teilhabe einschließlich möglicher Alternativen zur geplanten Versorgung,

- Zielsetzungen im Hinblick auf Behandlung/Therapie, Behinderungsausgleich, Förderung von Funktionen, Aktivitäten und Teilhabe,

- ausführliche Begründung der medizinischen Notwendigkeit einer Hilfsmittelversorgung oder deren Nicht-Notwendigkeit.

Vor der eigentlichen klinisch biomechanischen Prüfung erfolgt eine ambulante ärztliche Eingangsuntersuchung mit einer Anamneseerhebung, die insbesondere der Ermittlung relevanter Kontextfaktoren dient. Nach der klinischen Beurteilung der Stumpfbeschaffenheit und der Einstufung in die Aktivitätsklasse kann ggf. die Testfähigkeit attestiert werden. Der Auftraggeber erhält darüber einen ausführlichen Bericht und entscheidet auf dieser Basis, ob die Indikationsprüfung durchgeführt werden soll.

\section{Ermittlung der Aktivitätsklasse}

Die Einteilung in die Aktivitätsklassen 0 bis 4 erfolgt mittels des Mobilitätserhebungsbogens der Deutschen Gesetzlichen Unfallversicherung (DGUV; Formtext M2200):

- AK 0 - nicht gehfähig: Prothese als kosmetischer Ausgleich, für ein mikroprozessorgesteuertes Gelenk nicht geeignet,

- AK 1 - Innenbereichsgeher: Prothese als Transferhilfe, kurze Gehstrecken im häuslichen Bereich,

Trauma Berufskrankh 2016 • [Suppl 4]: 18:S359-S363 DOI 10.1007/s10039-016-0131-z

(c) Springer-Verlag Berlin Heidelberg 2016

\section{S. Simmel · S. Drisch · P. Augat · V. Bühren}

\section{Indikationsprüfung neuer Prothesenpassteile bei Oberschenkelamputierten}

\section{Zusammenfassung}

Auf das Gehen, Stehen und Treppensteigen ausgelegte Beinprothesen sind Körperersatzstücke, die dem Ersatz des fehlenden Körperteils und dessen ausgefallenen Funktionen dienen. Beim unmittelbaren Behinderungsausgleich gilt das Gebot eines möglichst weitgehenden Ausgleichs des Funktionsdefizits. Das Ziel der Versorgung ist letztendlich ein „Gleichziehen mit einem gesunden Menschen". Insbesondere die Entwicklung elektronischer Steuerungskonzepte in Prothesenkniegelenken ermöglicht einen weitergehenden Behinderungsausgleich, auf den Versicherte einen Anspruch haben, wenn er sich umfassend auf den Alltag auswirkt. Bei den gesetzlichen Unfallversicherungen besteht ein steigender Bedarf nach Überprüfung bzw. Stellung der Indikation für moderne mikroprozessorgesteuerte Prothesenpassteile. Der Kostenträger soll durch die Indikationsprüfung die Möglichkeit erhalten, dem amputierten Unfallverletzten das tatsächlich "geeignete Mittel" zur Verfügung zu stellen und die notwendige
Qualitätssicherung durchzuführen, um die hohen Kosten moderner Prothetik zu rechtfertigen. Nicht zuletzt muss die Versorgung auch wirtschaftlich sein. Hierfür ist der Nachweis eines objektivierbaren wesentlichen Gebrauchsvorteils durch das neue Hilfsmittel erforderlich. Im Rahmen einer mehrtägigen stationären Indikationsprüfung erfolgt eine umfangreiche Testung im Ganglabor und im Gehparcours zunächst mit der vorhandenen Prothetik. Nach der Probeversorgung mit dem zu testenden Passteil und einer Gebrauchsschulung werden die Tests mit dem neuen Prothesenpassteil wiederholt. Der Vergleich der Testergebnisse ermöglicht eine fundierte Aussage hinsichtlich der medizinischen Notwendigkeit einer Versorgung mit neuen Prothesenpassteilen, die auf jahrelanger Erfahrung, standardisierten Testverfahren und objektiven Testergebnissen basiert.

Schlüsselwörter

Amputation · Hilfsmittel · Aktivitätsklasse · Kosten · Indikationsprüfung

\section{Testing the indications for new prosthetic components in transfemoral amputees}

\section{Abstract}

Leg prostheses designed for normal walking, standing and climbing stairs are prostheses that serve as the direct replacement of the missing body part and its absent features. The disability should be compensated as far as possible. The aim of treatment is ultimately to be on an equal par with a healthy person. In particular, the development of electronic control concepts in prosthetic knee joints allows an extended compensation of the disability, which the insured persons are entitled to claim if it comprehensively impacts on everyday life. There is an increasing need for the statutory accident insurance to check the indications of modern microprocessorcontrolled prosthetic components, partly due to the rising costs. With the testing the insurance companies have the possibility of providing the amputee with a really appropriate means and to perform the necessary quality assurance, also in order to justify the high costs of modern prosthetics. Last but not least the treatment also has to be economically sound. For this purpose, the proof of a measurable significant advantage by using the new tools is required. During a hospital stay an extensive gait analysis and testing in a walking course is first performed with the existing prosthetics. After a test fitting with the new prosthetic part and instructions in use, the tests are repeated with the new prosthesis. The comparison of test results allows a professionally founded statement regarding the medical necessity of a provision with new prosthetic components based on years of experience, standardized test methods and objective test results.

\section{Keywords}

Amputation - Medical aid - Activity level . Costs $\cdot$ Indication test 
Tab. 1 Bewertungskriterien zur Überprüfung des funktionellen Zugewinns

\begin{tabular}{|llll}
\hline Prüfkriterium & Biomechanik & Klinik & $\begin{array}{l}\text { Subjektive Ein- } \\
\text { schätzung }\end{array}$ \\
\cline { 2 - 4 } & $\begin{array}{l}\text { Instrumentelle } \\
\text { Ganganalyse }\end{array}$ & $\begin{array}{l}\text { Standardisierte Tests } \\
\text { im Gehparcours/ } \\
\text { Gehschule }\end{array}$ & Patient \\
\hline Sicherheit & $(\mathrm{X})$ & $\mathrm{X}$ & $\mathrm{X}$ \\
\hline Entlastung & $\mathrm{X}$ & $(\mathrm{X})$ & $\mathrm{X}$ \\
\hline $\begin{array}{l}\text { Geteilte Aufmerk- } \\
\text { samkeit }\end{array}$ & - & $\mathrm{X}$ & $\mathrm{X}$ \\
\hline Geschwindigkeit & - & $\mathrm{X}$ & $\mathrm{X}$ \\
\hline Kraftaufwand & $(\mathrm{X})$ & $\mathrm{X}$ & $\mathrm{X}$ \\
\hline Gangbild & $\mathrm{X}$ & $\mathrm{X}$ & $\mathrm{X}$ \\
\hline Gehhilfe & - & $\mathrm{X}$ & $\mathrm{X}$ \\
\hline
\end{tabular}

- AK 2 - eingeschränkter Außenbereichsgeher: Stufen können überwunden werden,

- AK 3 - uneingeschränkter Außenbereichsgeher: die Geschwindigkeit kann in größerem Umfang variiert werden,

- AK 4 - uneingeschränkter Außenbereichsgeher mit besonders hohen Ansprüchen an die Mobilität, z. B. aufgrund sportlicher Aktivitäten.

Als Kriterium für die Aufnahme in eine Aktivitätsklasse wird das Erreichen einer Mindestpunktzahl vorausgesetzt.

Nach der ärztlichen Untersuchung erfolgen eine orthopädietechnische Erfassung und Überprüfung der bisherigen Prothesenversorgung, insbesondere eine Kontrolle der Passform des Prothesenschaftes und der Funktion der Prothesenpassteile.

Zur klinisch biomechanischen Prüfung werden nur Versicherte zugelassen, wenn keines der folgenden Ausschlusskriterien zutrifft:

- Zuordnung zu Aktivitätsklasse 0 oder 1 ,

- Prothesenschaft nicht passfähig,

- Belastbarkeit deutlich reduziert (z. B. instabile Angina pectoris),

- Prothesenerstversorgung,

- mangelnde Kooperationsbereitschaft.

\section{Kriterien des funktionellen Zugewinns}

Ziel der weiteren Prüfung ist die Feststellung des "funktionellen Zugewinns“ durch die geplante neue Versorgung. Da- bei setzt sich der funktionelle Zugewinn aus folgenden 7 Kriterien zusammen [3]:

- Sicherheit - Erhöhung der Standund Gangsicherheit: Das Kriterium Sicherheit umfasst die Möglichkeiten des alternierenden Treppabgehens und des Gehens auf unebenem Untergrund sowie das Bewältigen von Rampen und schiefen Ebenen (• Abb. 1).

- Entlastung - Entlastung der gesunden Gegenseite: Eine stärkere Belastung, optimal eine Vollbelastung der Prothese als Standbein, führt zur gewünschten Entlastung der Gegenseite und wirkt dem üblichen Bewegungsmuster mit einer langen Schrittweite und kurzen Standphase der Prothesenseite entgegen.

- Geteilte Aufmerksamkeit - Integration der Prothese in das Körperschema mit dadurch ermöglichter geteilter Aufmerksamkeit: Der Prothesenträger muss sich während des Gehens nicht ausschließlich auf die Prothese konzentrieren, kleinere Aufgaben können gleichzeitig ausgeführt werden (• Abb. 2).

- Geschwindigkeit - Variation der Geschwindigkeit: Variation der Geschwindigkeit umfasst zum einen die Möglichkeit, verschiedene Gangarten auszuführen, zum anderen auch das plötzliche Stehenbleiben auf Zuruf.

- Kraftaufwand - Verringerung des Kraftaufwandes: Die Verringerung des für den Gangzyklus benötigten Kraftaufwandes wird von Amputierten positiv als weniger anstrengendes und komfortableres Gehen wahrgenommen.

- Gangbild - Harmonisierung des Gangbildes: Bei Prothesenträgern ist das Gangbild häufig asymmetrisch. Die Harmonisierung des Gangbildes und die Integration der Prothese in das Körperschema stellen deshalb ebenfalls einen funktionellen Zugewinn dar.

- Gehhilfe - Reduktion der Hilfsmittel: Durch die Neuversorgung können vorher verwendete Gehhilfen, wie z. B. Unterarmgehstützen, weggelassen werden.

\section{Ablauf}

Nach Absprache mit dem Kostenträger und Einholung der Kostenübernahme wird der Proband stationär einbestellt. Die Indikationsprüfung neuer Prothesenpassteile findet im Rahmen einer i.d. R. 5-tägigen komplexen stationären Rehabilitation in der BGU Murnau statt. Voraussetzungen für die Durchführung der Testverfahren sind eine ausreichende körperliche Belastbarkeit, reizlose Stumpfverhältnisse, ein gut eingelaufener und passender Schaft sowie ein optimal eingestelltes neues Passteil.

Wesentliches Merkmal der klinisch biomechanischen Untersuchungen ist die vergleichende Testung des zu erprobenden Passteils gegen das bisher genutzte Passteil. Wenn möglich, wird nur ein Passteil getauscht, z. B. das Kniepassteil, während eine Änderung der Fußpassteile nicht erfolgt. Hierdurch soll ein direkter Vergleich - unabhängig von anderen Einflussgrößen - gewährleistet werden.

Nach Anreise des Probanden (in der Regel montags) findet ein ärztliches Aufnahmegespräch statt. Hierbei wird nochmals die Testfähigkeit überprüft. Zunächst erfolgt die erste Ganganalyse mit der bisherigen Prothese im Ganglabor des Institutes für Biomechanik. Dabei werden mittels Kraftmessplatten die Bodenreaktionskräfte ermittelt und über am Körper angebrachte Marker Bewegungsabläufe analysiert und dokumentiert (-Abb. 3). Im Anschluss erfolgt der physiotherapeutische klinische Test im Gehparcours und der Gehschule. Hier werden die Belastungsverteilung, 
das Gehen in der Ebene, die Möglichkeit von Geschwindigkeitswechsel, das Gehen auf verschiedenen Untergründen sowie Schrägen und Treppen - auch unter erschwerten Bedingungen („geteilte Aufmerksamkeit") - getestet. Eine wesentliche Rolle spielt auch die subjektive Einschätzung des Prothesenträgers, die standardisiert erfasst wird.

Nach der Testung mit dem bisherigen System (Tag 1), erfolgt die Versorgung mit dem neuen Passteil, das für einige Tage (Tag 2-4) intensiv im Rahmen der Amputiertengehschule, der medizinischen Trainingstherapie und Einzelphysiotherapie sowie bei Bedarf unter externen und/oder häuslichen Bedingungen erprobt wird. Am Tag 5 werden die abschließenden Tests (gleiche Testbatterie wie am Tag 1) mit dem neuen Passteil wiederholt.

\section{Auswertung und Indikations- stellung}

Die Prüfkriterien werden sowohl aus ganganalytischer als auch klinischer Sicht einzeln bewertet und die Testergebnisse zu Beginn und am Ende verglichen (•Tab. 1). Hieraus ergibt sich der funktionelle Zugewinn und damit, ob ein Gebrauchsvorteil im Alltag durch das neue Prothesenpassteil erwartet werden kann.

Wir gehen davon aus, dass ein Prothesenträger von einer neuen Versorgung „profitiert“, wenn er mindestens 4 Kriterien des funktionellen Zugewinns erfüllt. Insbesondere die „Sicherheit“ ist ein wichtiger Prädiktor für die Indikationsstellung. Wie von Kuhr [4] gezeigt, ermöglichen auch bereits 4 „Leitkriterien“ die Indikationsstellung:

1. Entlastung der Gegenseite,

2. erhöhte Sicherheit,

3. Harmonisierung des Gangbildes,

4. Ermöglichung von geteilter Aufmerksamkeit.

Von diesen 4 Leitkriterien sollten entweder die beiden ersten oder die beiden letzten Kriterien gleichzeitig einen $\mathrm{Zu}$ gewinn aufweisen (• Abb. 4).

Die Indikation zur Versorgung mit dem neuen Passteil wird gestellt, wenn ein funktioneller Zugewinn in mindes- tens 4 der 7 Prüfkriterien oder in mindestens 2 „Leitkriterien“ nach Kuhr (entsprechend Abb.4) sowohl objektiv (klinisch und biomechanisch) als auch subjektiv feststellbar ist.

\section{Fazit für die Praxis}

- Beim unmittelbaren Behinderungsausgleich gilt das Gebot eines möglichst weitgehenden Ausgleichs des Funktionsdefizits durch Beinprothesen.

- Die Entwicklung elektronischer Steuerungskonzepte in Prothesenkniegelenken ermöglicht einen weitergehenden Behinderungsausgleich, auf den Versicherte einen Anspruch haben, wenn er sich umfassend auf den Alltag auswirkt.

- Der Nachweis eines objektivierbaren wesentlichen Gebrauchsvorteils durch das neue Hilfsmittel ist für eine Verordnung erforderlich.

- Im Rahmen einer mehrtägigen stationären Indikationsprüfung erfolgt eine umfangreiche Testung. Der Vergleich der Testergebnisse zwischen alter und neuer Prothese ermöglicht eine fundierte Aussage hinsichtlich der medizinischen Notwendigkeit einer Versorgung mit neuen Prothesenpassteilen.

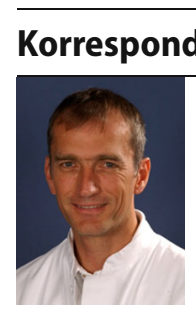

Dr. S. Simmel

BG-Unfallklinik Murnau Professor-Küntscher-Str. 8, 82418 Murnau, Deutschland stefan.simmel@bgumurnau.de

\section{Einhaltung ethischer Richtlinien}

Interessenkonflikt. S. Simmel, S. Drisch, P. Augat und V. Bühren geben an, dass kein Interessenkonflikt besteht.

Dieser Beitrag beinhaltet keine von den Autoren durchgeführten Studien an Menschen oder Tieren.

Alle Patienten, die über Bildmaterial oder anderweitige Angaben innerhalb des Manuskripts zu identifizieren sind, haben hierzu ihre schriftliche Einwilligung gegeben. Im Falle von nicht mündigen Patienten liegt die Einwilligung eines Erziehungsberechtigten oder des gesetzlich bestellten Betreuers vor.

\section{Literatur}

1. Gemeinsame Richtlinien der Verbände der Unfallversicherungsträger über die Hilfsmittelversorgung im Bereich der gesetzlichen Unfallversicherung (§31 Abs. 2 Satz 2 SGB VII), Stand: 1. Juli 2011. www.dguv.de Zugriff: 25. Februar 2016

2. Richtlinie des Gemeinsamen Bundesauschusses über die Verordnung von Hilfsmitteln in der vertragsärztlichen Versorgung (Hilfsmittel-Richtlinie), Stand: 17.7.2014, in Kraft getreten am 29.10.2014

3. Endbericht zum Prüfauftrag "Festlegung von Indikationen für eine Versorgung mit dem ,C-Leg Kniegelenksystem der Fa. Otto Bock, Duderstadt" für das Bundesministerium für Arbeit und Soziales - Stand: 30. März 2006, Klinische Prüfstelle für orthopädische Hilfsmittel, Universitätsklinikum Münster

4. Kuhr K (2008) Multivariate Analyse des funktionellen Zugewinns bei Kniegelenkssystemen (Diplomarbeit), Technische Universität Dortmund 